\title{
Phase coexistence and negative thermal expansion in the triple perovskite iridate $\mathrm{Ba}_{3} \mathrm{CoIr}_{2} \mathrm{O}_{9}$
}

\author{
Charu Garg, ${ }^{1}$ Antonio Cervellino, ${ }^{2}$ and Sunil Nair ${ }^{1, *}$ \\ ${ }^{1}$ Department of Physics, Indian Institute of Science Education and Research, Dr. Homi Bhabha Road, Pune 411008, India \\ ${ }^{2}$ Swiss Light Source, Paul Scherrer Institute, CH-5232 Villigen, Switzerland
}

(Received 9 October 2020; revised 24 February 2021; accepted 5 April 2021; published 16 April 2021)

\begin{abstract}
The anomalous thermal expansion in a layered $3 d-5 d$ based triple perovskite iridate $\mathrm{Ba}_{3} \mathrm{CoIr}_{2} \mathrm{O}_{9}$ is investigated using high resolution synchrotron diffraction. Below the magnetostructural transition at $107 \mathrm{~K}$, the onset of antiferromagnetic order is associated with a monoclinic distortion of the hexagonal structure. Deeper within the magnetically ordered state, a part of the monoclinic phase distorts even further, and both these structural phases co-exist down to the lowest measured temperatures. We observe negative thermal expansion in this phase co-existence regime, which appears to be intimately connected to the temperature driven relative fractions of these monoclinic phases. The significant NTE observed in this system could be driven by magnetic exchange striction and is of relevance to a number of systems with pronounced spin orbit interactions.
\end{abstract}

DOI: 10.1103/PhysRevMaterials.5.044405

\section{INTRODUCTION}

Negative thermal expansion (NTE) - the counterintuitive contraction of solids on heating-has been the focus of extensive experimental and theoretical investigations for many years [1-4]. By virtue of their structural diversity and chemical flexibility, the transition metal oxides have proven to be a fertile playground for uncovering new candidates which exhibit this phenomena [5-9]. From the structural perspective, this phenomena is feasible in systems where the crystallographic framework consists of strongly bonded polyhedra units (also called rigid unit modes) [10], and here the NTE occurs as a consequence of the transverse vibrations of these units. NTE can also arise due to underlying electronic and magnetic phenomena [11-13]. A significant advance in this field was the observation of giant NTE in $\mathrm{ZrW}_{2} \mathrm{O}_{8}$ [14,15], which then paved the way for this phenomena to be discovered in a number of other transition metal oxides. A variety of structural classes like the zeolites, perovskites, and its variants, as well as spinels, are now known to exhibit NTE [12,16-19].

The $5 d$ based transition metal oxides, especially iridates, have recently gained considerable attention given the diverse electronic, magnetic, and structural ground states they exhibit. A number of exotic phenomena ranging from spin-orbit liquids, charge ordering, superconductivity, Weyl semimetals, and heavy fermions have now been reported [20]. Owing to the extended nature of the iridium $5 d$ orbitals, several energy scales like the onsite Hubbard interaction $U$, Hund's coupling $\mathrm{J}_{H}$, the SOC $\lambda$, the crystal field $\Delta$, and the hopping energy $t$ become comparable, and a complex interplay between these competing scales results in their multifunctional attributes. Compared to their $3 d$ counterparts where spin (S) and orbital angular momentum (L) are considered to be good quantum

\footnotetext{
*sunil@iiserpune.ac.in
}

numbers, strong spin orbit coupling in the $5 d$ compounds leads to the formation of $\mathrm{J}_{\text {eff }}$ states, also called pseudospin states [21,22]. Many interesting ground states have been attributed to the presence of the pseudospin lattice (PSL) coupling which is two orders of magnitude higher compared to the manganites [23]. In compounds with strong spin orbit coupling, the high sensitivity of the ground state to local lattice distortions modifies the spin-phonon dynamics that are reflected through changes in the structural parameters and the magnetization response $[20,24]$. The presence of pseudospin in iridates has generated much curiosity and recent studies on single and bilayer Ruddlesden-Popper iridates of the form $\mathrm{Sr}_{n+1} \mathrm{Ir}_{n} \mathrm{O}_{3 n+1}(n=1,2$, inf $)$ bear interesting results [23,2529]. These studies were focused on the $J_{\text {eff }}=1 / 2$ state for iridium where $\mathrm{IrO}_{6}$ octahedra are connected via corners in the $a-b$ plane and disconnected via a SrO layer along the $c$ axis. The pseudospin states coupled to lattice distortions have been speculated to promote tetragonal to orthorhombic distortions in the $\mathrm{J}_{\text {eff }}=1 / 2 \mathrm{Sr}_{2} \mathrm{IrO}_{4}$ system and anisotropic negative thermal expansion in the bilayered $\mathrm{Sr}_{3} \mathrm{Ir}_{2} \mathrm{O}_{7}[23,25,26]$.

A relatively recent addition to the strongly correlated iridates are the layered triple perovskites of the form $A_{3} X \mathrm{Ir}_{2} \mathrm{O}_{9}$ where $A$ is an alkaline earth metal, and $X$ can be an alkali metal, alkali earth metal, $3 d$ transition metal, or a lanthanide. Most of these systems adopt a hexagonal $6 \mathrm{H}$ triple perovskite in which the crystal structure is based on $\mathrm{XO}_{6}$ octahedra connected via corners to face sharing $\operatorname{Ir}_{2} \mathrm{O}_{9}$ dimers. Given the complex geometry of corner and face sharing octahedra, the lattice is frustrated and extremely susceptible to perturbations. It has been observed that this frustration is typically released in the form of a magnetic and structural phase transformation [30-34]. In spite of the structural diversity and chemical flexibility offered by these iridates, a functionality which has not been seriously explored pertains to the feasibility of NTE in these systems.

Here, we report structural and magnetic investigations of $\mathrm{Ba}_{3} \mathrm{CoIr}_{2} \mathrm{O}_{9}$, a $3 d-5 d$ based member of this triple perovskite 
family where we propose that the pseudospin lattice coupling (PSL), formed as a consequence of strong spin orbit entanglement, leads to a crystal structure transformation and magnetic exchange striction driven negative thermal expansion. This system exhibits a structural transition at $107 \mathrm{~K}$, accompanied by the onset of canted antiferromagnetic order. Unlike any other reported triple perovskite, this system shows two structural transitions with clear evidence of phase coexistence below $70 \mathrm{~K}$. Moreover, we observe signs of magnetic stress driven negative thermal expansion at low temperatures in both these coexisting phases. Such a correlation between the stress and the crystallographic structure is possible due to the open framework structure and flexible network intrinsic to this system, where open spaces facilitate lattice deformations and octahedral rotations. This stress, which has a magnetic origin, could be important in a number of related systems with large spin-orbit interactions. We note that the nature of PSL coupling in the case of the nominally $\mathrm{J}_{\text {eff }}=0$ systems is still an open question, and our results could hold useful pointers on its relevance in the strongly correlated iridates.

\section{EXPERIMENTAL DETAILS}

Polycrystalline specimens of $\mathrm{Ba}_{3} \mathrm{CoIr}_{2} \mathrm{O}_{9}$ were synthesized using the solid state synthesis route, and details of the synthesis and characterization have been reported previously [35]. Synchrotron x-ray diffraction measurements were performed using the Materials Science (MS) X04SA beam line (wavelength $0.56526 \AA$ ) at the Swiss Light Source (SLS, PSI Switzerland [36]). The powder sample was filled in a $0.3 \mathrm{~mm}$ capillary and the experiments were carried out in the temperature range $4.2 \mathrm{~K}-295 \mathrm{~K}$. The crystallographic structure was analyzed by the Rietveld method using the FullProf refinement program [37]. The structures shown in the paper are drawn using Vesta [38]. Magnetization measurements were performed using a Quantum Design (MPMS-XL) SQUID magnetometer.

\section{RESULTS AND DISCUSSION}

$\mathrm{Ba}_{3} \mathrm{CoIr}_{2} \mathrm{O}_{9}$ crystallizes in an aristotype $6 \mathrm{H}$-type $\mathrm{BaTiO}_{3}$ hexagonal $\left(\mathrm{PG}_{3} / \mathrm{mmc}\right)$ structure at room temperature, consistent with the previously reported triple perovskites [30,39]. This structure constitutes of two $\mathrm{IrO}_{6}$ octahedra sharing a face along the $c$ axis, with these $\mathrm{Ir}_{2} \mathrm{O}_{9}$ dimers being connected by corner sharing $\mathrm{CoO}_{6}$ octahedra. The structure of the triple perovskites of the form $A_{3} B B_{2}^{\prime} O_{9}$ depends on the relative radii of $A, B$, and $B^{\prime}$ ions [40]. As the size of the $A$ cation increases, the $\mathrm{BO}_{6}$ and $\mathrm{B}^{\prime} \mathrm{O}_{6}$ octahedra rotate in order to minimize the lattice energy. This tilting of octahedra then modifies bond lengths and bond angles, as a consequence of which the effective crystallographic symmetry is reduced. The room temperature lattice parameters of $\mathrm{Ba}_{3} \mathrm{CoIr}_{2} \mathrm{O}_{9}$ as obtained from Rietveld refinement of $\mathrm{x}$-ray diffraction data are $\mathrm{a}=$ $\mathrm{b}=5.7639 \AA, \mathrm{c}=14.2949 \AA$, and $\alpha=\beta=90^{\circ}, \gamma=120^{\circ}$. On lowering temperature, the structure transforms from the hexagonal $P 6_{3} / m m c$ to a monoclinic $C 2 / c$ symmetry at the magnetostructural transition temperature of $107 \mathrm{~K}$, with this symmetry being retained down to $80 \mathrm{~K}$. On further lowering of the temperature, the system partially transforms to an even

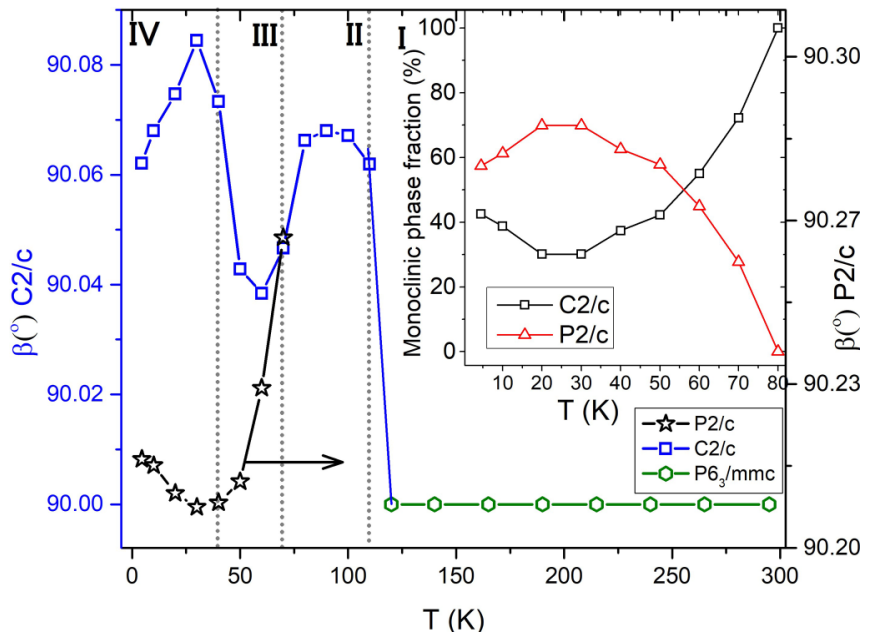

FIG. 1. Main panel: Temperature dependence of the monoclinic angle $\beta$ for the $P 6_{3} / m m c(\bigcirc), C 2 / c(\square)$ and $P 2 / c$ ( $\star$ ) phases of $\mathrm{Ba}_{3} \mathrm{CoIr}_{2} \mathrm{O}_{9}$. The variation of phase fraction of the monoclinic $P 2 / c$ and $C 2 / c$ phases with temperature is depicted in the inset.

lower symmetry $(P 2 / c)$, with both these structurally disparate phases coexisting down to the lowest measured temperatures. The details of the structural parameters for all the phases are given in Ref. [35].

The evolution of relative ratios of both the monoclinic phases with temperature is shown in the inset of Fig. 1. Unless explicitly depicted, the error bars for all the crystallographic parameters deduced from analysis of diffraction data are of the same order (or smaller) than the symbol size used. As the temperature is reduced, the $C 2 / c$ phase gradually converts to the lower $P 2 / c$ symmetry. Interestingly, close to 40 $\mathrm{K}\left(T_{p}\right)$, this phase ratio is reversed and a part of the system slowly transforms back to the higher $C 2 / c$ monoclinic symmetry. The evolution of these phases can be clearly observed in $\beta$ which is the angle between the crystallographic $a$ and $c$ axes, as depicted in the main panel of Fig. 1. The plot can be divided into four regions. Region I corresponds to the (paramagnetic) hexagonal phase where $\beta$ remains constant based on symmetry constraints. In region II, there is a sharp increase in $\beta$ because of the change in symmetry at the magnetostructural transition. Region III corresponds to the phase coexistence regime, and as the $C 2 / c$ phase transforms to $P 2 / c$, the $\beta$ value sharply increases till the phase crossover temperature $\left(T_{p}\right)$ is reached. As soon as a maximum tilt angle of $90.084^{\circ}\left( \pm 0.002^{\circ}\right)$ is achieved, the phase reversal is triggered. This corresponds to region IV, where $\beta$ decreases with a concomitant increase in the relative fraction of the $C 2 / c$ phase. A similar trend is also observed for the lower symmetry $P 2 / c$ phase, with temperature dependence of $\beta$ and the phase fraction being inversely correlated to each other.

On careful observation, these regions are also clearly demarcated in the temperature dependence of the volume, as is shown in Fig. 2(a). A steep rise in the volume at $107 \mathrm{~K}$ marks the onset of AFM order and a magnetostructural transition as confirmed by synchrotron and neutron diffraction. A dashed vertical bar at $70 \mathrm{~K}$ indicates the onset of second phase transition concomitant with the onset of a phase coexistence region. Interestingly, the monoclinic $P 2 / c$ phase shows an anomalous 


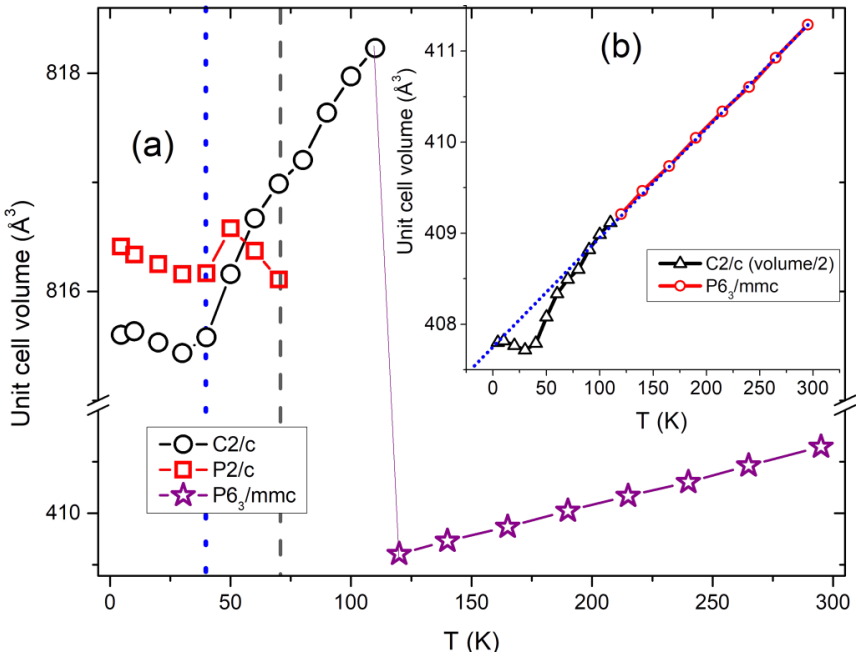

FIG. 2. (a) Temperature dependence of volume as determined for $P 6_{3} / m m c(\star), C 2 / c(\bigcirc)$, and $P 2 / c(\square)$. The vertical bars at $70 \mathrm{~K}$ and $40 \mathrm{~K}$ indicate the onset of phase coexistence and the phase crossover temperature $T_{P}$ respectively. (b) The reduced volume for $C 2 / c$ is plotted for comparison and a strong upturn is observed close to $40 \mathrm{~K}$. (c) Variation of the coefficient of volume thermal expansion $\alpha_{V}$ for the $C 2 / c$ and $P 2 / c$ phases.

behavior where the volume increases as the temperature is lowered. This negative thermal expansion is observed right from the phase transformation at $70 \mathrm{~K}$ with a discontinuity at the phase crossover temperature $T_{p}$ of $40 \mathrm{~K}$. Since the magnetostructural transition from the hexagonal $P 6_{3} / m m c$ to the monoclinic $C 2 / c$ phase at $107 \mathrm{~K}$ is accompanied by unit cell doubling, the reduced volume of the $P 6_{3} / m m c$ phase and the volume of the $C 2 / c$ phase are plotted against temperature in Fig. 2(b) for an easy comparison. A subtle change in the unit cell volume at the structural transition $(107 \mathrm{~K})$ is followed by a marked upturn in the volume at $40 \mathrm{~K}$, as is also depicted in the main panel. This clearly indicates the presence of negative thermal expansion in the $C 2 / c$ phase as well below the phase crossover temperature $T_{p}$.

The crossover from positive to negative thermal expansion results from the balance between two competitive factors: anharmonic phonon vibration and magnetic exchange striction, with each contributing to positive and negative thermal expansion, respectively. The highest values of the coefficient of volume thermal expansion $\alpha_{V}[=(1 / \mathrm{V}) d \mathrm{~V} / d T]$ for the $P 2 / c$ and $C 2 / c$ phases of $\mathrm{Ba}_{3} \mathrm{CoIr}_{2} \mathrm{O}_{9}$ are -1.21 ppm $\mathrm{K}^{-1}$ and $-0.8 \mathrm{ppm} \mathrm{K}^{-1}$, respectively, which is of the same order as that reported in many prototypical NTE materials [41,42]. For instance, the prototypical NTE system $\mathrm{ZrW}_{2} \mathrm{O}_{8}$ was reported to exhibit $\alpha_{V}=-2.7 \mathrm{ppm} \mathrm{K}^{-1}$, albeit in a larger temperature range.

The temperature dependence of the $c / a$ ratio for all the structural phases is depicted in Fig. 3, and significant change is observed just below the magnetostructural transition. This is indicative of a structural distortion arising due to magnetic exchange striction-which in antiferromagnetic transition metal oxides results in the generation of a strain field aimed at increasing the magnetic exchange energy [43]. The lattice parameter $c$ for both the monoclinic phases is depicted in

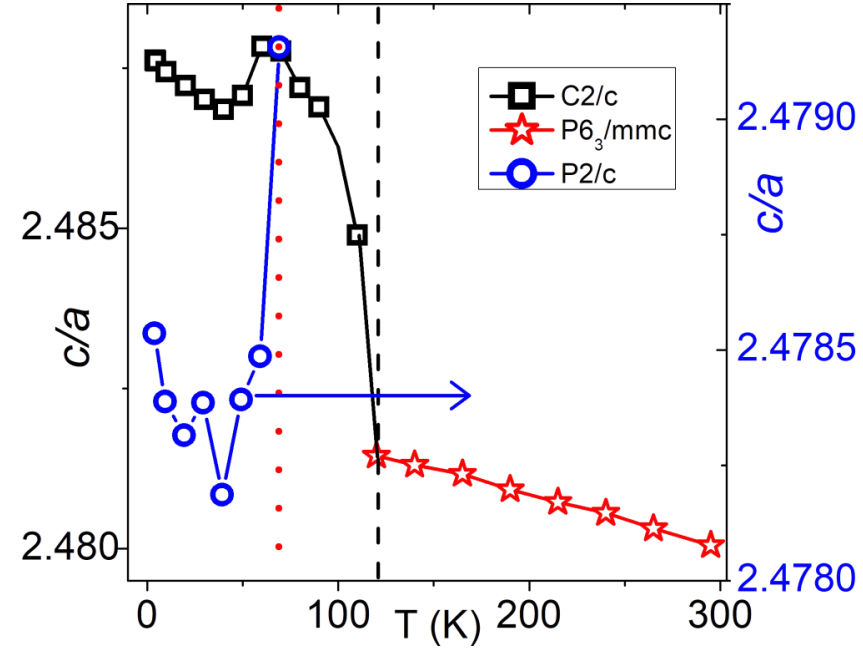

FIG. 3. Temperature dependence of the $c / a$ ratio for all the structural phases encountered in $\mathrm{Ba}_{3} \mathrm{CoIr}_{2} \mathrm{O}_{9}$. The dashed bar indicates the onset of magnetostructural transition and the dotted line represents the start of the phase coexistence regime.

Fig. 4(a). For the $P 2 / c$ phase, it increases as a function of decreasing temperature for the entire temperature range, with a discontinuity at $T_{p}$. On the other hand, in the $C 2 / c$ phase, a negative slope is attained only at $T_{p}$. Interestingly, the lattice parameter $a$ shows a NTE in the $P 2 / c$ phase and a positive

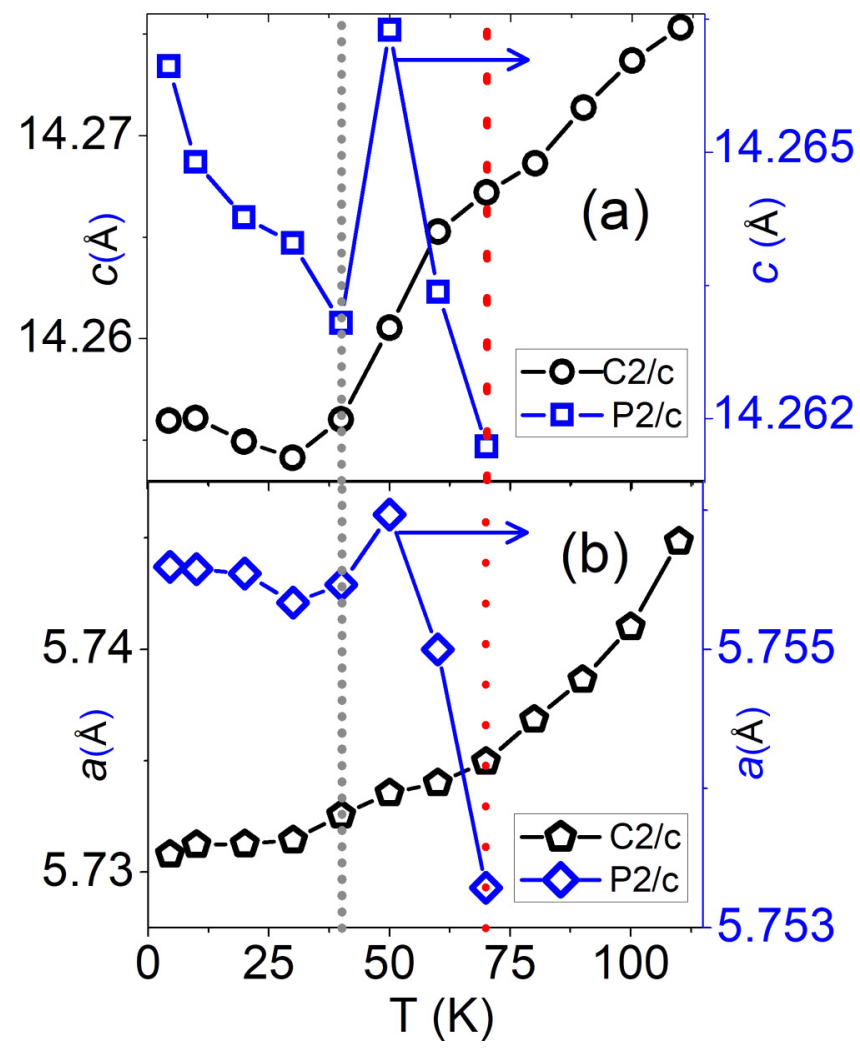

FIG. 4. Variation of lattice parameters $c$ and $a$ with temperature is depicted in (a) and (b), respectively. The dashed and dotted line indicate the onset of phase coexistence and the phase crossover temperature, respectively. 
thermal expansion (PTE) in the $C 2 / c$ phase throughout the temperature range as shown in Fig. 4(b). The contrasting temperature dependence of the lattice parameters is indicative of anisotropic thermal expansion and also reinforces the critical influence which the lattice parameter $c$ has on the NTE in this material. Such strong anisotropic negative thermal expansion in the bilayered iridates has been reported to arise as a consequence of strong pseudospin lattice coupling [23], which is reflected in the form of a phonon anomaly due to an increase in the interlayer spacing below the magnetic transition. The fact that none of the other triple perovskites reported until this date are known to exhibit NTE suggests that this phenomena is driven by the phase coexistence and phase reversal which is unique to the $\mathrm{Ba}_{3} \mathrm{CoIr}_{2} \mathrm{O}_{9}$ system.

There are broadly two types of NTE [2]: one which arises solely due to a structural origin - often termed as conventional NTE. In these systems, the structural framework consists of strongly bonded polyhedra units called RUMs (rigid unit modes) [10] and NTE occurs as a consequence of the transverse vibrations of these units. Due to its structural origin, they are generally present over a broad temperature range. The other type of NTE is driven by phase transitions, with underlying phenomena like charge transfer [44], ferroelectric order [45], magnetic and orbital ordering [46], and giant magnetocaloric effect [47] being responsible. Interestingly, many oxides like $\mathrm{LaMnO}_{3}[7,8], \mathrm{SrRuO}_{3}[9]$, and $\mathrm{AlFeO}_{3}[12]$ exhibit this type of NTE. Prerequisites for observing negative thermal expansion by the transverse thermal motion of oxygen include: (i) an open framework structure with oxygen in a twofold coordination, (ii) strong metal-oxygen bonds to avoid significant thermal expansion of the bond length, and (iii) rigid polyhedra which are free to tilt back and forth with little or no change in shape. The monoclinically distorted triple perovskite structure of $\mathrm{Ba}_{3} \mathrm{CoIr}_{2} \mathrm{O}_{9}$ appears to be susceptible to distortions allowed by the RUM model due to the flexible network and open framework structure intrinsic to this symmetry. Interestingly, these rotations of the $\mathrm{IrO}_{6}$ and $\mathrm{CoO}_{6}$ octahedra also allow for the overall monoclinic symmetry to be preserved while transforming to a lower space group. Figure 5(a) displays the change in the Ir-O-Ir bond angles in the face sharing dimer for the $C 2 / c$ phase. Although the Ir-O2-Ir angle remains relatively rigid below the magnetostructural transition, the Ir-O1-Ir angle has a strong temperature dependence. As soon as it reaches the maxima of octahedral tilting at the phase crossover temperature $\left(T_{P}\right)$, the octahedra are buckled, as is reflected in the sharp decrease in the Ir-O1-Ir angle. A similar trend is seen in Co-O-Ir for the $P 2 / c$ monoclinic phases as shown in Fig. 5(b), where a distinct change of slope corresponding to the buckling of the octahedra is observed. This correlation between the NTE and the octahedral distortions is seen even more clearly in the temperature dependence of the tilt angle of the $\mathrm{CoO}_{6}$ octahedra which sits at the corners of the unit cells in both the $C 2 / c$ and the $P 2 / c$ phases, as is depicted in Fig. 6(b). In the monoclinic $C 2 / c$ phase, this angle is initially invariant and increases on approaching the phase coexistence regime as shown in Fig. 6(a). The stabilization of the monoclinic $P 2 / c$ phase partially relieves this rotation, and this quantity is seen to be inversely correlated in both the phases. This is an indicator of how the lattice strain in one of the phases is relieved by the stabilization of the other.

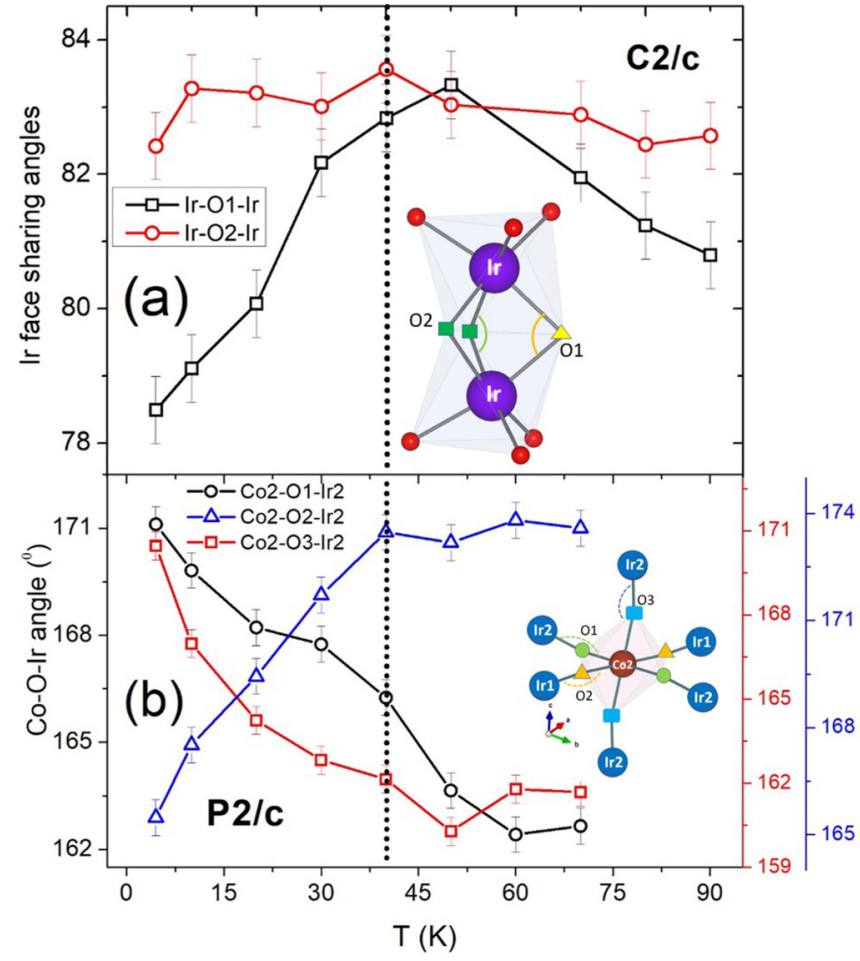

FIG. 5. (a) Main panel: Thermal evolution of face sharing Ir-O-Ir angles within the Ir dimer depicted pictorially in the inset. O1( $\mathbf{\Delta})$ and $\mathrm{O} 2(\boldsymbol{\square})$ represent the face sharing oxygen. (b) Main panel: Temperature variation of the three unique Co-O-Ir angles made by cobalt and iridium in the $C 2 / c$ and $P 2 / c$ phases. Inset: A schematic representation of the cobalt octahedra connected via corner to iridium. The vertical dotted line represents the phase crossover temperature $T_{P}$.

Moreover, signatures of this phase coexistence is also seen as a hump in the dc magnetization, which is also plotted in the same graph for clarity. Though there is no evidence of a low temperature magnetic transition in this system, it is evident that the magnetization is modulated by the crystallographic structure, indicating the intimate connection between the two.

Our prior neutron diffraction and first principles calculations indicate that the spins are predominantly aligned along the crystallographic $c$ axis in this system [35]. With the structural distortions and magnetization appearing to be intimately coupled, it is not surprising that the major lattice change is observed along this direction. Figure 7 depicts the hysteresis in the magnetization as measured in the field cooled cooling (FCC) and the field cooled warming (FCW) protocols in the phase coexistence regime. This indicates the first order nature of this transition, which is associated with formation of a new phase $P 2 / c$ inside the monoclinic $C 2 / c$ phase which is stabilized below the magnetostructural transition at $107 \mathrm{~K}$. This intimate coupling between the magnetism and NTE is reminiscent of the large magnetostriction and NTE observed in the frustrated spinel $\mathrm{ZnCrSe}_{4}$ [16]. It was suggested that the NTE in that system arose as a consequence of a highly frustrated lattice, with exchange striction being the driving mechanism. Similarly in $\mathrm{Sr}_{3} \mathrm{Ir}_{2} \mathrm{O}_{7}$, a phonon anomaly was observed due to the coupling between the $c$-axis antiferromagnetic pseudospin long range order and the out of plane phonon mode. This 

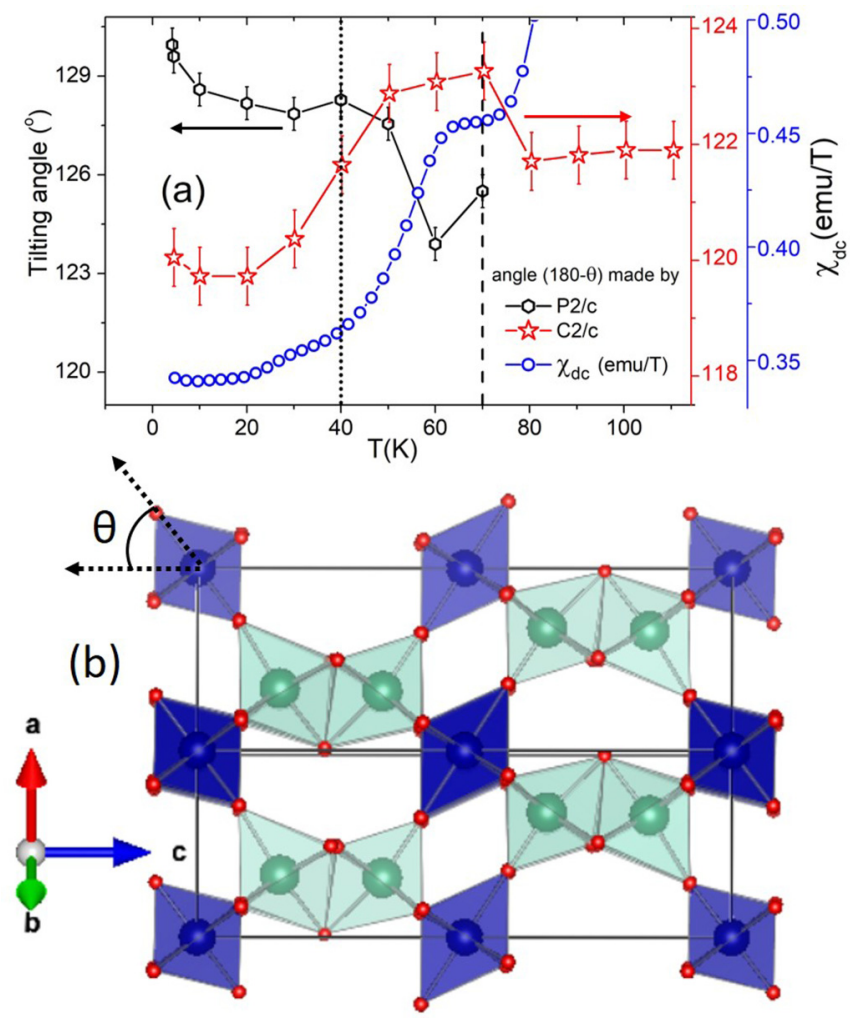

FIG. 6. Top panel: Temperature-dependent dc magnetization data measured in a magnetic field of 100 Oe for $\mathrm{Ba}_{3} \mathrm{CoIr}_{2} \mathrm{O}_{9}$. Red and black curves indicate the tilt angle of $\mathrm{CO}_{6}$ octahedra for $C 2 / c$ and $P 2 / c$, respectively, as shown in the schematic below. The $\mathrm{CO}_{6}$ octahedra sits at the corner and $\theta$ is the angle formed by the apical oxygen with the $c$ axis.

was accompanied by an increase in interplanar spacing below the magnetic transition and a negative thermal expansion was reported as a consequence of this pseudospin lattice coupling [23]. We note that in $\mathrm{Ba}_{3} \mathrm{CoIr}_{2} \mathrm{O}_{9}$ which orders magnetically

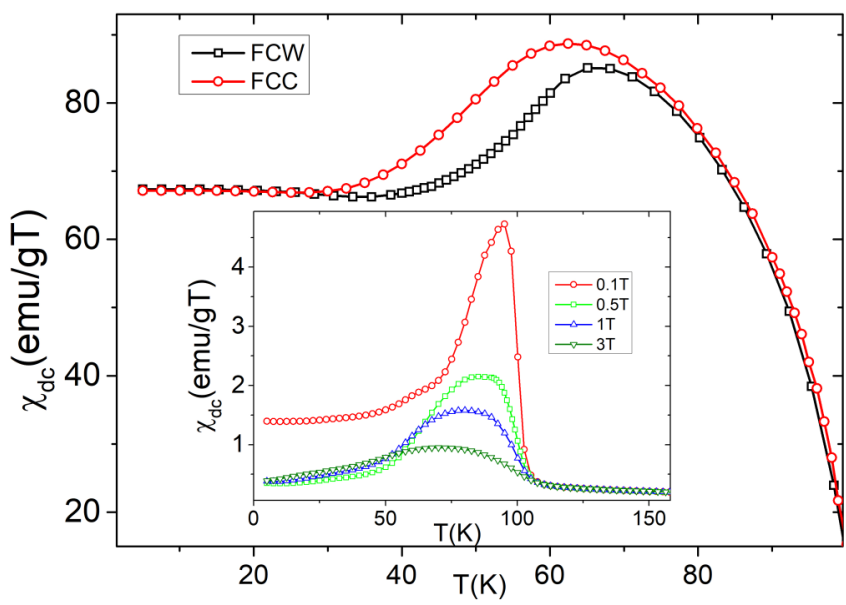

FIG. 7. Main panel: Temperature-dependent dc magnetization data measured under a magnetic field of 1000e under field-cooled warming (FCW) and field-cooled cooling (FCC) protocols. Inset: Temperature dependence of zero field-cooled (ZFC) dc magnetization data of $\mathrm{Ba}_{3} \mathrm{CoIr}_{2} \mathrm{O}_{9}$ at different magnetic fields. at $107 \mathrm{~K}$, a fit to the high temperature magnetization data gave a Curie-Weiss temperature $\left(\theta_{\mathrm{CW}}\right)$ of $6.35 \mathrm{~K}$, which is clearly suggestive of mixed ferroantiferromagnetic interactions [35]. This was also confirmed by measurements of $\mathrm{MH}$ isotherm, where a small loop opening - typical of ferri- or weak ferromagnetic systems-was observed. The magnetic structure in the $C 2 / c$ phase was deduced to be a canted antiferromagnet, with the main antiferromagnetic component along the $c$ axis. The increase in the interplanar spacing along the $c$ axis leads to modifications in the orbital overlap between the $\mathrm{Co}^{+2}\left(3 d^{7}\right) / \mathrm{Ir}^{+5}\left(5 d^{4}\right)$ and $\mathrm{O}^{-2}(2 p)$ orbitals, as reflected by the changes in the magnetization which traces the octahedral tilting down to the lowest measured temperatures. The concordant locking of the octahedral rotation to the canted magnetic moments has been previously observed in the iridates even in the presence of local noncubic lattice deformations [29]. Though the antiferromagnetic magnetic structure in the $P 2 / c$ phase remains to be ascertained, it is likely that it only differs in the extent of the canting angle. The absence of negative thermal expansion in the ruthenate analog $\mathrm{Ba}_{3} \mathrm{CoRu}_{2} \mathrm{O}_{9}$ indicates that this phenomena is intrinsic to the iridates where strong coupling between pseudo spin and lattice results in complex ground states. An interesting aspect of $\mathrm{Ba}_{3} \mathrm{CoIr}_{2} \mathrm{O}_{9}$ pertains to the temperature regime in which NTE is observed. For instance, in systems like $\mathrm{Ca}_{2} \mathrm{RuO}_{4}$ [13], $\mathrm{Pb}(\mathrm{Ti}, \mathrm{V}) \mathrm{O}_{3}$ [48], and $\mathrm{Mn}_{3} \mathrm{ZnN}$ [49], the NTE is driven by an electronic or magnetic phase transition and is observed (or has its onset) in the transition region. Conversely, in the $\mathrm{ZnCrSe}_{4}$ system, NTE was observed predominantly in the paramagnetic regime (starting from $T>3 \times T_{N}$ ) down to the magnetic transition temperature, owing to short range spin fluctuations characteristic to geometrically frustrated magnets. In contrast to these cases, the NTE in $\mathrm{Ba}_{3} \mathrm{CoIr}_{2} \mathrm{O}_{9}$ is observed deep within the AFM state and is intimately related to a phase coexistence between the two disparate monoclinic phases.

This situation is similar to that reported earlier in the rare earth vanadates of the form $R \mathrm{VO}_{3}$ (with $R=\mathrm{Sm}, \mathrm{Ho}, \mathrm{Yb}$, Pr, or Y) [50,51], where competing orbitally ordered phases coexist as a consequence of the competition between the lattice strain arising out of octahedral tilting and that due to exchange striction. We speculate that a similar mechanism is at play here and that the temperature evolution of the structurally disparate monoclinic phases in $\mathrm{Ba}_{3} \mathrm{CoIr}_{2} \mathrm{O}_{9}$ arises as a consequence of the interplay between exchange striction, relative energy difference between the $C 2 / c$ and $P 2 / c$ phases, as well as octahedral rotations in each phase. On traversing the magnetostructural transition from the high temperature paramagnetic $\left(\mathrm{Pb}_{3} / \mathrm{mmc}\right)$ state, we first encounter the $C 2 / c$ phase which appears to be energetically most favorable. However, increasing exchange striction as a function of reducing temperature strains the lattice, which in turn is partially relieved by a conversion of a part of the $C 2 / c$ phase to the lower symmetry $P 2 / c$ phase starting from $70 \mathrm{~K}$. The relative energy difference between these monoclinic phases appears to be dynamic, and below the crossover temperature, a part of the $P 2 / c$ phase converts back to the higher symmetry $C 2 / c$ phase. However, this alone does not compensate for the exchange striction induced strain, and a part of it is accommodated by the octahedral rotations in both these disparate phases which then manifests itself in the form of NTE in both of them. 
This interplay between competing phases is also likely to make the ground state amenable to external perturbations. A pronounced magnetic field dependence of the NTE has been previously observed in $\mathrm{ZnCrSe}_{4}$ [16] where the balance between the AFM and FM states in the frustrated magnetic spinels can be easily tuned with the application of a magnetic field $[16,52]$. Though both the coexisting phases in the case of $\mathrm{Ba}_{3} \mathrm{CoIr}_{2} \mathrm{O}_{9}$ are AFM, it is quite likely that perturbations like pressure or magnetic fields can tilt the balance between them. Our zero field cooled measurements at different applied magnetic fields as shown in the inset of Fig. 7 indicate that the broad hump signifying the region of phase coexistence gets suppressed and shifts to even low temperature values as a function of increasing magnetic fields. Magnetic field dependent measurements of $\mathrm{x}$-ray diffraction and magnetostriction are likely to throw more light on the evolution and dynamics of these co-existing phases.

\section{CONCLUSIONS}

In summary, we report on the existence of a low temperature regime of negative thermal expansion in a new $3 d-5 d$ based triple perovskite iridate $\mathrm{Ba}_{3} \mathrm{CoIr}_{2} \mathrm{O}_{9}$. This unusual temperature dependence of the volume is intimately related to the coexistence of two closely related monoclinic phases and the temperature dependence of their relative phase fractions. The underlying driving mechanism appears to be the pseudospin lattice coupling induced antiferromagnetic exchange striction, which precipitates a phase conversion from a monoclinic $C 2 / c$ phase to a more distorted $P 2 / c$ phase as a function of decreasing temperatures. Below a phase crossover temperature of $40 \mathrm{~K}$, the phase ratios of these monoclinic phases inverts, with the remainder of the strain being accommodated by octahedral distortions which manifests itself in the form of a negative thermal expansion in both these phases. This work opens up opportunities in exploring NTE in intrinsically layered systems which are characterized by strong spin orbit interactions. Investigation of the PSL coupling can be of immediate significance to systems with strong spin-orbit coupling and could be generic to the strongly frustrated iridates.

\section{ACKNOWLEDGMENTS}

S.N. acknowledges DST India for support through the DST Nanomission Thematic Unit Program, SR/NM/TP-13/2016. C.G. and S.N. thank the Department of Science and Technology, India (SR/NM/Z-07/2015) for financial support and Jawaharlal Nehru Centre for Advanced Scientific Research (JNCASR) for managing the project.
[1] G. D. Barrera, J. A. O. Bruno, T. H. K. Barron, and N. L. Allan, J. Phys.: Condens. Matter 17, R217 (2005).

[2] K. Takenaka, Frontiers in Chemistry 6, 267 (2018).

[3] K. Takenaka, Sci. Technol. Adv. Mater. 13, 013001 (2012).

[4] W. Miller, C. Smith, D. Mackenzie, and K. Evans, J. Mater. Sci. 44, 5441 (2009).

[5] J. Chen, L. Hu, J. Deng, and X. Xing, Chem. Soc. Rev. 44, 3522 (2015).

[6] T. Chatterji, P. F. Henry, R. Mittal, and S. L. Chaplot, Phys. Rev. B 78, 134105 (2008).

[7] J. G.-M. noz, M. Suaaidi, and C. Ritter, Phys. B: Condens. Matter 234-236, 854 (1997), proceedings of the First European Conference on Neutron Scattering.

[8] Q. Huang, A. Santoro, J. W. Lynn, R. W. Erwin, J. A. Borchers, J. L. Peng, and R. L. Greene, Phys. Rev. B 55, 14987 (1997).

[9] T. Kiyama, K. Yoshimura, K. Kosuge, Y. Ikeda, and Y. Bando, Phys. Rev. B 54, R756 (1996).

[10] M. T. Dove, V. Heine, and K. D. Hammonds, Mineral. Mag. 59, 629 (1995).

[11] K. Takenaka, M. Ichigo, T. Hamada, A. Ozawa, T. Shibayama, T. Inagaki, and K. Asano, Sci. Technol. Adv. Mater. 15, 015009 (2014).

[12] R. Saha, A. Shireen, A. Bera, S. N. Shirodkar, Y. Sundarayya, N. Kalarikkal, S. Yusuf, U. V. Waghmare, A. Sundaresan, and C. Rao, J. Solid State Chem. 184, 494 (2011).

[13] K. Takenaka, Y. Okamoto, T. Shinoda, N. Katayama, and Y. Sakai, Nat. Commun. 8, 14102 (2017).

[14] T. A. Mary, J. S. O. Evans, T. Vogt, and A. W. Sleight, Science 272, 90 (1996).

[15] G. Ernst, C. Broholm, G. Kowach, and A. Ramirez, Nature (London) 396, 147 (1998).
[16] J. Hemberger, H.-A. K. von Nidda, V. Tsurkan, and A. Loidl, Phys. Rev. Lett. 98, 147203 (2007).

[17] G. Pokharel, A. F. May, D. S. Parker, S. Calder, G. Ehlers, A. Huq, S. A. J. Kimber, H. S. Arachchige, L. Poudel, M. A. McGuire, D. Mandrus, and A. D. Christianson, Phys. Rev. B 97, 134117 (2018).

[18] L. A. Villaescusa, P. Lightfoot, S. J. Teat, and R. E. Morris, J. Am. Chem. Soc. 123, 5453 (2001).

[19] D. A. Woodcock, P. Lightfoot, L. A. Villaescusa, M.-J. DíazCabañas, M. A. Camblor, and D. Engberg, Chem. Mater. 11, 2508 (1999).

[20] J. G. Rau, E. K.-H. Lee, and H.-Y. Kee, Annu. Rev. Condens. Matter Phys. 7, 195 (2016).

[21] B. J. Kim, H. Jin, S. J. Moon, J.-Y. Kim, B.-G. Park, C. S. Leem, J. Yu, T. W. Noh, C. Kim, S.-J. Oh, J.-H. Park, V. Durairaj, G. Cao, and E. Rotenberg, Phys. Rev. Lett. 101, 076402 (2008).

[22] G. Khaliullin, Prog. Theor. Phys. Suppl. 160, 155 (2005).

[23] L. L. Hu, M. Yang, Y. L. Wu, Q. Wu, H. Zhao, F. Sun, W. Wang, R. He, S. L. He, H. Zhang, R. J. Huang, L. F. Li, Y. G. Shi, and J. Zhao, Phys. Rev. B 99, 094307 (2019).

[24] G. Cao and P. Schlottmann, Rep. Prog. Phys. 81, 042502 (2018).

[25] J. Porras, J. Bertinshaw, H. Liu, G. Khaliullin, N. H. Sung, J.-W. Kim, S. Francoual, P. Steffens, G. Deng, M. M. Sala, A. Efimenko, A. Said, D. Casa, X. Huang, T. Gog, J. Kim, B. Keimer, and B. J. Kim, Phys. Rev. B 99, 085125 (2019).

[26] H. Liu and G. Khaliullin, Phys. Rev. Lett. 122, 057203 (2019).

[27] H. Gretarsson, N. H. Sung, M. Höppner, B. J. Kim, B. Keimer, and M. Le Tacon, Phys. Rev. Lett. 116, 136401 (2016).

[28] S. Boseggia, H. C. Walker, J. Vale, R. Springell, Z. Feng, R. S. Perry, M. M. Sala, H. M. Rønnow, S. P. Collins, 
and D. F. McMorrow, J. Phys.: Condens. Matter 25, 422202 (2013).

[29] D. H. Torchinsky, H. Chu, L. Zhao, N. B. Perkins, Y. Sizyuk, T. Qi, G. Cao, and D. Hsieh, Phys. Rev. Lett. 114, 096404 (2015).

[30] H. D. Zhou, A. Kiswandhi, Y. Barlas, J. S. Brooks, T. Siegrist, G. Li, L. Balicas, J. G. Cheng, and F. Rivadulla, Phys. Rev. B 85, 041201(R) (2012).

[31] S. A. J. Kimber, M. S. Senn, S. Fratini, H. Wu, A. H. Hill, P. Manuel, J. P. Attfield, D. N. Argyriou, and P. F. Henry, Phys. Rev. Lett. 108, 217205 (2012).

[32] K. Yokota, N. Kurita, and H. Tanaka, Phys. Rev. B 90, 014403 (2014).

[33] M. Lee, E. S. Choi, J. Ma, R. Sinclair, C. R. D. Cruz, and H. D. Zhou, J. Phys.: Condens. Matter 28, 476004 (2016).

[34] K. M. Ranjith, K. Brinda, U. Arjun, N. G. Hegde, and R. Nath, J. Phys.: Condens. Matter 29, 115804 (2017).

[35] C. Garg, D. Roy, M. Lonsky, P. Manuel, A. Cervellino, J. Müller, M. Kabir, and S. Nair, Phys. Rev. B 103, 014437 (2021).

[36] P. R. Willmott, D. Meister, S. J. Leake, M. Lange, A. Bergamaschi, M. Böge, M. Calvi, C. Cancellieri, N. Casati, A. Cervellino, Q. Chen, C. David, U. Flechsig, F. Gozzo, B. Henrich, S. Jäggi-Spielmann, B. Jakob, I. Kalichava, P. Karvinen, J. Krempasky, A. Lüdeke, R. Lüscher, S. Maag, C. Quitmann, M. L. Reinle-Schmitt, T. Schmidt, B. Schmitt, A. Streun, I. Vartiainen, M. Vitins, X. Wang, and R. Wullschleger, J. Synchrotron Radiat. 20, 667 (2013).

[37] J. Rodriguez-Carvajal, An Introduction to the Programme FULLPROF (Laboratoire Leon Brillouin, CEA-CNRS, Saclay, France, 2001).

[38] K. Momma and F. Izumi, J. Appl. Cryst. 44, 1272 (2011).
[39] U. Treiber, S. Kemmler-Sack, and A. Ehmann, Z. Anorg. Allg. Chem. 487, 189 (1982).

[40] T. Sakamoto, Y. Doi, and Y. Hinatsu, Journal of Solid State Chemistry France 179, 2595 (2006).

[41] K. Takenaka and H. Takagi, Appl. Phys. Lett. 87, 261902 (2005).

[42] I. Yamada, K. Tsuchida, K. Ohgushi, N. Hayashi, J. Kim, N. Tsuji, R. Takahashi, M. Matsushita, N. Nishiyama, T. Inoue, T. Irifune, K. Kato, M. Takata, and M. Takano, Angew. Chem., Int. Ed. 50, 6579 (2011).

[43] A. Filippetti and N. A. Hill, Phys. Rev. Lett. 85, 5166 (2000).

[44] M. Azuma, W.-t. Chen, H. Seki, M. Czapski, S. Olga, K. Oka, M. Mizumaki, T. Watanuki, N. Ishimatsu, N. Kawamura et al., Nat. Commun. 2, 347 (2011).

[45] J. Chen, F. Wang, Q. Huang, L. Hu, X. Song, J. Deng, R. Yu, and X. Xing, Sci. Rep. 3, 2458 (2013).

[46] T. F. Qi, O. B. Korneta, S. Parkin, J. Hu, and G. Cao, Phys. Rev. B 85, 165143 (2012).

[47] F. Hu, F. Shen, J. Hao, Y. Liu, J. Wang, J. Sun, and B. Shen, Frontiers in Chemistry 6, 438 (2018).

[48] Z. Pan, J. Chen, X. Jiang, L. Hu, R. Yu, H. Yamamoto, T. Ogata, Y. Hattori, F. Guo, X. Fan et al., J. Am. Chem. Soc. 139, 14865 (2017).

[49] T. Hamada and K. Takenaka, J. Appl. Phys. 109, 07E309 (2011).

[50] R. Saha, F. Fauth, V. Caignaert, and A. Sundaresan, Phys. Rev. B 95, 184107 (2017).

[51] M. H. Sage, G. R. Blake, C. Marquina, and T. T. M. Palstra, Phys. Rev. B 76, 195102 (2007).

[52] J. Hemberger, T. Rudolf, H.-A. Krug von Nidda, F. Mayr, A. Pimenov, V. Tsurkan, and A. Loidl, Phys. Rev. Lett. 97, 087204 (2006). 